\title{
Effect of Individual and Combined Salinity and High Temperature Stress during Germination Stage of Different Wheat (Triticum aestivum L.) Genotypes
}

\author{
Neelambari ${ }^{1 *}$, Ajay Kumar Singh ${ }^{2}$ and Shailesh Kumar ${ }^{3}$ \\ Department of Botany and Plant Physiology, Dr. Rajendra Prasad Central Agricultural \\ University, Pusa-848125, Bihar, India \\ Corresponding author
}

\begin{tabular}{|l|}
\hline Key w o r d s \\
Salinity, High \\
temperature, \\
Combined stress
\end{tabular}

A B S T R A C T

Global warming and soil salinity drastically reduced the productivity of cereals including wheat which is the most important food crop next to rice. A laboratory experiment was conducted with three replications to screen forty six winter wheat (Triticum aestivum L.) genotypes for individual salinity and high temperature stress and their combined effect at germination and early seedling growth stage. The seeds were subjected to two levels of salinity stress i.e. 4 and $8 \mathrm{dSm}^{-1}$ with salt combination of $\mathrm{NaCl}: \mathrm{CaCl}_{2}: \mathrm{Na}_{2} \mathrm{SO}_{4}$ in a ratio of $7: 2: 1$. The high temperature treatment was given by transferring the seeding in the incubator at temperature $37 \pm 2{ }^{\circ} \mathrm{C}$. The physiological parameters i.e. germination percentage, root length, shoot length, seedling length, root/shoot length ratio, vigour index I and SPAD value were measured in 10 days old seedling. The result indicated that germination percentage; root/shoot length ratio, root/shoot fresh weight Ratio, root/shoot dry weight Ratio and SPAD unit value were decreased most severely under combined second salinity level and high temperature stress when compared to ambient condition. However, root/shoot length ratio sowed an irregular pattern i.e. at individual and combined lower salinity level and high temperature stress more wheat genotypes showed increase in root/shoot length ratio, while rest showed decrease in the same. However, under individual and combined higher salinity level and high temperature stress more genotype showed reduction in the same parameter. Based on the physiological parameters, the genotype KRL-1-4, KRL-19 and HD-2733 was found to be most tolerant and HI-1563, HD 2329 and HT-8 were the most susceptible to salinity at germination stage and early seedling stage. In conclusion, there is genetic variability among winter wheat genotypes that can be used in breeding programs to improve winter wheat yield under combined high temperature and salinity stress conditions.

\section{Introduction}

In the field, crops are normally exposed to a combination of one or two or multiple abiotic stresses. Critical abiotic stresses that crops are commonly exposed to include drought, high temperature, salinity, and lack of nutrients. These abiotic stresses are often interrelated, 
either individually or in combination, they cause morphological, physiological, biochemical, and molecular changes that adversely affect plant growth and productivity, and ultimately yield. Although salt stress and heat shock have been extensively studied but little is known about their combined effects on plants. Many studies have described the response mechanisms of plants to salinity and heat applied individually, recent studies revealed that the response of plants to a combination of two different stresses is specific and cannot be deduced from the stresses applied individually (Rivero et al., 2014). Salinity and high temperatures are the major environmental factors that limit wheat productivity. Climate change model forecast that in the future inland salinity and short or long episodes of high temperature can decrease the crop productivity. As a cool season crop, wheat grows best when temperatures are in a range of 21 to $25{ }^{\circ} \mathrm{C}$; however, it requires different temperatures at different stages of plant growth and development. At a temperature below or above the optimum temperature, germination of the seed decreases (Bowden et al., 2008). A temperature increase of $3-4{ }^{\circ} \mathrm{C}$ could cause crop yields to fall by $15-35 \%$ in Africa and Asia (Ortiz et al., 2008). Heat stress is thus a common problem in many wheat-growing regions worldwide. Soil salinity is a global eco-threat to sustainable agriculture and is also increasing over the time. Seed germination, one of the most critical phases in plant life, is greatly affected by salinity (Abo-Kassem, 2007), which either induces a state of dormancy at low levels or completely inhibits germination at higher levels (Iqbal et al., 2006). It may cause decrease in some physiological aspects of wheat during germination such as speed of germination, the length, fresh and dry weight of the root and shoot, chlorophyll and carotenoids contents (Turkyilmaz, 2012). To cope with environmental stresses, plants can develop adaptation strategies. There are two distinct strategies used by plants to deal with different abiotic stresses. The first strategy is stress avoidance, which allows the plant to avoid the exposure of plant systems to the stress factors by excluding those factors or their effects from plant systems. The second strategy is stress tolerance, which is the ability of the plant to sustain plant function with the presence of stressed conditions (Touchette et al., 2007).

\section{Materials and Methods}

The present study was done in laboratory condition, to identify the contrasting set of wheat genotypes among the forty six wheat genotypes i.e. HT-150, KRL-19, KRL-213, KRL210， KRL1-4， HD-2888， DBW-16, DBW-14, CSW 16, C-306, HW-2045, HI1563, BRW-934, PBW-373, HD-2985, WR544, HD-2643, K-68, HD-3059, PBW-65, KACHU\#1, HT-142, HT-138, HD-2733, HD2329, HT-140, RAJ-4120, HP-1939, HTEM, HT-20, BRW-8708, HT-147, HT-22, GS2027, GS-2021, HT-8, GS-2008, GS-1010, GS-1001， HT-114， GS-2028， HD-2967, BAAZ, K-8027, GS-1020 and GS-2007, based on changes in physiological traits under study, subjected to individual and combined salinity and high temperature stress. Three replications of 20 wheat seeds were sown in each sterilized petri plate lined with three layers of filter paper for germination. For salt stress treatments, saline solution of composition $\mathrm{Nacl}: \mathrm{CaCl}_{2}: \mathrm{Na}_{2} \mathrm{SO}_{4}$ (7:2:1) and concentration 4 (salinity level $1, \mathrm{~S}_{1}$ ) and $8 \mathrm{ds} / \mathrm{m}$ (salinity level 2, $\mathrm{S}_{2}$ ) were used as irrigation solution. For high temperature treatment the petri dishes were kept in incubator at temperature $37 \pm$ $2^{\circ} \mathrm{C}$ and for combined salinity and high temperature stress, salinity stressed plant (induced by application of saline solution of EC 4 and $8 \mathrm{ds} / \mathrm{m}$ ) was shifted to elevated temperature $\left(37 \pm 2{ }^{\circ} \mathrm{C}\right)$ in incubator. Control plants were grown under ambient/ unstressed 
conditions $\left(25 \pm 2{ }^{\circ} \mathrm{C}\right)$. The physiological parameters recorded were germination percentage $(\%)$, Root length $(\mathrm{cm})$, Shoot length $(\mathrm{cm})$, Seedling length $(\mathrm{cm})$, Root /shoot length ratio and SPAD value were recorded on $10^{\text {th }}$ day after germination. Vigour index- I was also recorded at $10^{\text {th }}$ day after germination as per procedure prescribed by Abdul-Baki and Anderson (1973). Data analysis was performed using the software "DSAASTAT" statistical software (Version 1.101). Mean separations were performed by Duncan's Multiple Range Test (DMRT) at $1 \%$ and 5\% level.

\section{Results and Discussion}

\section{Germination percentage}

Considering the effect of high temperature $(\mathrm{T})$, salinity level $1\left(\mathrm{~S}_{1}\right)$, combined salinity level 1 and high temperature $\left(S_{1} T\right)$, salinity level $2\left(S_{2}\right)$ and combined salinity level 2 and high temperature $\left(\mathrm{S}_{2} \mathrm{~T}\right)$ stress, it was observed that germination percentage decreased remarkably with the different stress treatments and it was at the tune of 7.4, 14.7, 18.9, 28.4 and $32 \%$ respectively, compared with control and when averaged across genotypes. Under control condition the genotype KRL-1-4 showed highest germination percentage however genotype HT-20 showed poorest germination percentage. High temperature overall had a significant negative effect on germination percentage in all wheat genotypes with maximum decrease in genotypes, HI1563, HD2985 and HT-8 however, high temperature showed no effect on the given parameter in genotypes KRL-19 and HD-2733 and a minimum reduction in genotype KRL-14, compared to control. Essemine et al., (2007) also showed similar result that low temperature reduced germination capacity and delayed germination percentage. Similar result was given by Nyachiro et al., (2002) in wheat. Similarly, salinity level 1 and salinity level 2 significantly reduced germination percentage in all wheat genotypes, however, maximum reduction in germination percentage was observed in genotypes, HI-1563, HT-8 and HD2985 and no effect was observed in genotypes KRL-19, HD-2733 and KRL-1-4, compared to control. Also the severity of reduction was more under $S_{2}$ level The result was in agreement with Biabani et al., (2013) in wheat. Similar result was reported by Akbarimoghaddam et al., (2011) and Datta et al., (2009) in wheat and Fuller et al., (2012) in rye. Combined salinity level1 and high temperature stress also, drastically decreased germination percentage with maximum decrease in genotypes HI-1563, HT-8 and HD2985 and minimum decrease in genotypes KRL-19, HD-2733 and KRL-1-4 when compared to optimum condition. This result was in agreement with Kamkar et al., 2002 in millet. However, combined salinity level 2 and high temperature stress also significantly decreased germination percentage and it was more pronounced then all other stress treatments. Among genotypes the reduction in germination percentage due to the given combined stress was highest in genotypes HD2985, HT-8 and HI-1563 and least reduction in genotypes KRL-19, HD-2733 and KRL-14. Hokmalipour (2015) showed similar result in cumin and fennel. Based on the result it was concluded that wheat genotypes KRL-19, HD2733 and KRL-1-4 were able to tolerate to some extent, the negative effect of individual and combined salinity and high temperature stress and was considered as tolerant however genotypes HD-2985, HT-8 and HI-1563 as susceptible. This may be due to the reason that under different stress condition there may be lack of optimum temperature require for proper germination, slow rate of water imbibitions, ionic toxicity, oxidative damage etc.

\section{Root/shoot length ratio}

Combination of different stress treatments i.e. $\mathrm{T}, \mathrm{S}_{1}, \mathrm{~S}_{1} \mathrm{~T}, \mathrm{~S}_{2}$ and $\mathrm{S}_{2} \mathrm{~T}$ exhibited an irregular 
pattern of increase and decrease in root/shoot length ratio and it was at the tune of 0.8, 0.6, $0.6,2$ and $6 \%$ respectively, compared with optimum condition and when averaged across genotypes. Under control condition genotype KRL- 19 showed largest root/shoot length ratio however, genotype GS-2008 showed smallest root/shoot length ratio. High temperature stress showed an irregular pattern of result i.e. in some genotypes the stress induced the parameter with the largest root/shoot length ratio was exhibited by KRL213 while, decrease the root/shoot length ratio in some other genotypes with lowest root/shoot length ratio was exhibited by HT20. High temperature caused negative effects on shoot and root growth (Du and Tachibana, 1994; Gur et al., 1972; Lyons et al., 2007; Xu and Huang, 2000a). Also Arai-Sanoh et al., (2010) studied similar effects of temperature on growth and root function in rice. A little reduction in root/shoot length ratio was observed in genotypes KRL-1-4, KRL-19 and HD-2733 for treatment $\mathrm{S} 1$ and $\mathrm{S}_{2}$. While, genotypes HT-8, HI1563 and HD-2985 showed a little increase in the root/shoot length ratio for treatment $\mathrm{S} 1$ while decrease in the same under treatment $\mathrm{S} 2$ with a percentage decrease of $1.8,1$ and $0.103 \%$ respectively. Hameed et al., (2008) also reported similar effects of $\mathrm{NaCl}$ salinity on the growth parameters (root and shoot length, root/shoot length ratio, root and shoot fresh and dry weight and root and shoot fresh and dry weight ratios). Treatments under combined stress $\left(\mathrm{S}_{1}\right.$ Tand $\left.\mathrm{S}_{2} \mathrm{~T}\right)$ severely decreased shoot length with maximum decrease in genotypes HI-1563, HT-8 and HD-2985 and minimum decrease in genotypes KRL-19, HD-2733 and KRL-1-4 for treatment $\mathrm{S}_{1} \mathrm{~T}$ and $\mathrm{S}_{2} \mathrm{~T}$ when compared to control. However, reduction in the shoot length was more pronounced in treatment $\mathrm{S}_{2} \mathrm{~T}$ then other stress treatments. The result reveled that, wheat genotypes KRL-19, HD-2733 and KRL-1-4 were considered as tolerant as they were able to maintain least reduction in the given parameters under different stress applied either individually and in combinations. However, genotypes HD2985, HT-8 and HI-1563 were considered as susceptible.

\section{Root/shoot fresh weight ratio}

Stress treatments i.e. $\mathrm{T}, \mathrm{S}_{1}, \mathrm{~S}_{1} \mathrm{~T}, \mathrm{~S}_{2}$ and $\mathrm{S}_{2} \mathrm{~T}$ exhibited progressive decrease in root/shoot fresh weight ratio and it was at the tune of 7.6, 13, 16.1, 19.8, and $26.8 \%$ respectively, compared with optimum condition and when averaged across genotypes. Under control condition genotype HD-2733 showed root/shoot fresh weight ratio however, genotype GS-2007 showed smallest root/shoot fresh weight ratio. High temperature stress showed a gradual decrease in the root/shoot fresh weight ratio while intensity of reduction was less in genotypes KRL-1-4, KRL-19 and HD-2733 while more in genotypes HI-1563, HD-2985 and HT-8. In the same way the negative effect of temperature on seedling growth of psyllium, marshmallow and fennel flower with regards to germination behavior and seedling growth characteristics (seedling length, fresh weight dry weight and there ratios) is well documented in Saba et al., (2014). For treatment $S 1$ and $S_{2}$, genotypes KRL-1-4, KRL-19 and HD-2733 showed least reduction in the parameter while, genotypes HT-8, HI-1563 and HD-2985 showed a highest reduction compared to optimum condition. Similarly, Moud and Maghsoudi (2008) observed that salt stress inhibited coleoptiles root/shoot fresh weight ratio. Similarly, treatments under combined stress $\left(\mathrm{S}_{1}\right.$ Tand $\left.\mathrm{S}_{2} \mathrm{~T}\right)$ severely decreased shoot length with maximum decrease in genotypes HI1563, HT-8 and HD-2985 and minimum decrease in genotypes KRL-19, HD-2733 and KRL-1-4 for treatment $\mathrm{S}_{1} \mathrm{~T}$ and $\mathrm{S}_{2} \mathrm{~T}$ when compared to control. However, reduction in the shoot length was more pronounced in treatment $\mathrm{S}_{2} \mathrm{~T}$ then other stress treatments. The 
result reveled that, wheat genotypes KRL-19, HD-2733 and KRL-1-4 were considered as tolerant as they were able to maintain least reduction in the given parameters under different stress applied either individually and in combinations. However, genotypes HD2985, HT-8 and HI-1563 were considered as susceptible.

\section{Root/shoot dry weight ratio}

Stress treatments i.e. $T, S_{1}, S_{1} T, S_{2}$ and $S_{2} T$ exhibited progressive decrease in root/shoot dry weight ratio and it was at the tune of 6.9, $12.2,19.8,21$, and $29.1 \%$ respectively, compared with optimum condition and when averaged across genotypes. Under control condition genotype KRL-19 showed root/shoot fresh weight ratio however, genotype HI-1536 showed smallest root/shoot dry weight ratio. High temperature stress showed a gradual decrease in the root/shoot fresh weight ratio while intensity of reduction was less in genotypes KRL-1-4, KRL-19 and HD-2733 while more in genotypes HI-1563, HD-2985 and HT-8. In the same way the negative effect of temperature on seedling growth of psyllium, marshmallow and fennel flower with regards to germination behavior and seedling growth characteristics (seedling length, fresh weight dry weight and there ratios) is well documented in Saba et al., (2014). For treatment $S 1$ and $S_{2}$, genotypes KRL-1-4, KRL-19 and HD-2733 showed least reduction in the parameter while, genotypes HT-8, HI-1563 and HD-2985 showed a highest reduction compared to optimum condition. Hameed et al., (2008) also reported similar effects of $\mathrm{NaCl}$ salinity on the growth parameters (root and shoot length, root/shoot length ratio, root and shoot fresh and dry weight and root and shoot fresh and dry weight ratios). Similarly, treatments under combined stress ( $\mathrm{S}_{1}$ Tand $\left.\mathrm{S}_{2} \mathrm{~T}\right)$ severely decreased shoot length with maximum decrease in genotypes HI-1563, HT-8 and
HD-2985 and minimum decrease in genotypes KRL-19, HD-2733 and KRL-1-4 for treatment $\mathrm{S}_{1} \mathrm{~T}$ and $\mathrm{S}_{2} \mathrm{~T}$ when compared to control. However, reduction in the shoot length was more pronounced in treatment $\mathrm{S}_{2} \mathrm{~T}$ then other stress treatments. The result reveled that, wheat genotypes KRL-19, HD-2733 and KRL-1-4 were considered as tolerant as they were able to maintain least reduction in the given parameters under different stress applied either individually and in combinations. However, genotypes HD-2985, HT-8 and HI1563 were considered as susceptible.

\section{SPAD Value}

The data indicated that SPAD value progressively decreased under different stress treatments i.e. $\mathrm{T}, \mathrm{S}_{1}, \mathrm{~S}_{1} \mathrm{~T}, \mathrm{~S}_{2}$ and $\mathrm{S}_{2} \mathrm{~T}$, it was observed that SPAD Value decreased remarkably with the different stress treatments and it was at the tune of 6, 10.1, 15.6, 20.6 and $24.4 \%$ respectively, compared with control and when averaged across genotypes. Under control condition the genotype KRL-1-4 showed highest SPAD Value however genotype HD-2985 showed poorest germination percentage. High temperature overall had a significant negative effect on SPAD Value in all wheat genotypes with maximum decrease in genotypes, HI-1563, HD-2985 and HT-8 however, high temperature showed minimum effect on the given parameter in genotypes KRL-19 and HD-2733 and KRL-1-4 compared to control. Similarly, Feng et al., (2014) showed that heat stress caused a loss of pigment content in flag leaves. Heat stress reduces plant photosynthetic capacity through metabolic limitations and oxidative damage to chloroplasts and chlorophyll pigments (Farooq et al., 2011). Similarly, salinity level 1 (S1) significantly reduced SPAD Value in all wheat genotypes, however, maximum reduction in the same was observed in genotypes, HI-1563, HT-8 and HD-2985 and a last reduction was 
observed in genotypes KRL-19, HD-2733 and KRL-1-4 compared to control. In a similar study on tolerance capacity of wheat and bean cultivars to salinity salt stress Radi et al., (2013) reported a marked reduction in photosynthetic pigments (chlorophyll and carotenoids content) with gradual rise of salt level. Shaddad et al., (2013) also reported similar result in wheat. Combined salinity level 1 and high temperature stress $\left(\mathrm{S}_{1} \mathrm{~T}\right)$ also, drastically decreased SPAD Value with maximum decrease in genotypes HI-1563, HT-8 and HD2985 and minimum decrease in genotypes KRL-19, HD-2733 and KRL-1-4 when compared to optimum condition. Combined stresses of high temperature and salinity decreased the photosynthetic rate in wheat due to decrease in pigmentation as reported by Ehtaiwesh et al., (2016). Similarly, greater reduction in SPAD Value was observed with salinity level $2\left(\mathrm{~S}_{2}\right)$ in all wheat genotypes, with maximum decrease in same was observed in genotypes, HI-1563, HT-8 and HD-2985 and least reduction in genotypes KRL-19, HD-2733 and KRL-1-4 compared to control. Similarly, Turkyilmaz (2012) revealed that salinity decreased chlorophyll and carotenoids contents of wheat under salinity stress condition. Combined salinity level2 and high temperature (S2T) stress also significantly decreased SPAD Value and it was more pronounced then other stress treatments. Among genotypes the reduction in SPAD Value due to the given combined stress was highest in genotypes HD-2985, HT-8 and HI-1563 (41.8, 29.0 and 41.6 respectively) and least reduction in genotypes KRL-19, HD-2733 and KRL-1-4 (13, 12.6 and $14.5 \%$ respectively). Becker et al., 2017 also studied the effects of combined soil salinity and high temperature on photosynthesis and growth of quinoa plants (Chenopodium quinoa). Thus wheat genotypes KRL-19, HD-2733 and KRL-1-4 were considered as tolerant as they were able to tolerate the different stress interactions and genotypes HD-2985, HT-8 and HI-1563 were considered as susceptible. Such result may be due to the increased activity of chlorophyllase enzyme and reduced activity of Rubisco enzyme.

In conclusion it was observed that soil salinity and high temperatures are the major factors limiting growth of wheat crop and the intensity of the detrimental effect was more pronounced under combined stress. There is a genetic variability among the winter wheat genotypes that can be used in breeding programs to improve winter wheat yield under combined high temperature and salinity stress conditions. On the basis of result the wheat genotypes KRL-19, HD-2733 and KRL-1-4 were considered as tolerant as they were able to tolerate the different stress interactions and genotypes HD-2985, HT-8 and HI-1563 were considered as susceptible.

\section{References}

Abo-Kassem EEM. (2007). Effects of salinity: Calcium interaction on growth and nucleic acid metabolism in five species of Chenopodiaceae. Turk. J. Bot, 31: 125-134.

Akbarimoghaddam, H., Galavi, M., Ghanbari, A., and Panjehkeh, N. (2011). Salinity effects on seed germination and seedling growth of bread wheat cultivars. Trakia journal of Sciences, 9(1): 43-50.

Arai-Sanoh, Y., Ishimaru, T., Ohsumi, A., and Kondo, M. (2010). Effects of soil temperature on growth and root function in rice. Plant production science, 13(3): 235-242.

Becker, V. I., Goessling, J. W., Duarte, B., Caçador, I., Liu, F., Rosenqvist, E., and Jacobsen, S. E. (2017). Combined effects of soil salinity and high temperature on photosynthesis and growth of quinoa plants 
(Chenopodium quinoa). Functional Plant Biology, 44(7): 665-678.

Biabani, A., Hamideh, H., and Mosarreza, V. T. (2013). Salinity effect of stress on germination of wheat cultivars. Inter $J$ Agric Food Sci Tech, 4: 263-268.

Bowden, P., N. Ferguson,. (2008). Germination and emergence. In: J. White and J. Edwards (Eds.). Wheat growth and development. State of New South Wales through NSW Department of Primary Industries, pp 10-27.

Datta, J. K., Nag, S., Banerjee, A., and Mondai, N. K. (2009). Impact of salt stress on five varieties of Wheat (Triticum aestivum L.) cultivars under laboratory condition. Journal of Applied Sciences and Environmental Management, 13(3).

Du, Y.C. and Tachibana, S. (1994). Effect of supraoptimal root temperature on the growth, root respiration and sugar content of cucumber plants. Sci. Hort, 58: 289-301.

Ehtaiwesh, A. F. A. (2016). Effects of salinity and high temperature stress on winter wheat genotypes. (Doctoral dissertation, Kansas State University).

Essamine, J., Ammar, S., and Bouzid, S. (2010). Impact of heat stress on germination and growth in higher plants: physiological, biochemical and molecular repercussion and mechanisms of defense. J. Biol. Sci, 10(6): 565-572.

Farooq, M., Bramley, H., Palta, J. A., and Siddique, K. H. (2011). Heat stress in wheat during reproductive and grainfilling phases. Critical Reviews in Plant Sciences, 30(6): 491-507.

Feng, B., Liu, P., Li, G., Dong, S. T., Wang, F. H., Kong, L. A., and Zhang, J. W. (2014). Effect of Heat Stress on the Photosynthetic Characteristics in Flag Leaves at the Grain-Filling Stage of
Different Heat-Resistant Winter Wheat Varieties. Journal of agronomy and crop science, 200(2): 143-155

Fuller, M. P., Hamza, J. H., Rihan, H. Z., and Al-Issawi, M. 2012. Germination of primed seed under $\mathrm{NaCl}$ stress in wheat. ISRN Botany 2012.

Gur, A., Bravado, B. and Mizirahi, Y. (1972). Physiological response of apple trees to supraoptimal root temperatures. Physiol. Plant, 27:130-138.

Hameed, A., Naseer, S., Iqbal, T., Syed, H., and Haq, M. A. (2008). Effects of $\mathrm{NaCl}$ salinity on seedling growth, senescence, catalase and protease activities in two wheat genotypes differing in salt tolerance. Pak. J. Bot, 40(3): 1043-1051.

Hokmalipour, S. (2015). Effect of Salinity and Temperature on Seed Germination and Seed Vigor Index of Chicory (Chichorium intynus L.), Cumin (Cuminium cyminium L.) and Fennel (Foeniculum vulgare). Indian Journal of Science and Technology, 8(35).

Iqbal M, Ashraf M, Jamil A., and ur-Rehman S. (2006). Does seed priming induce changes in the levels of some endogenous plant hormones in hexaploid wheat plants under salt stress. J. Integr. Plant Biol, 48: 181189.

Kamkar, B., Vakili, S., and Mirizadeh, A. (2009). Effects of salinity and temperature on germination of three millet varieties. Journal of Seed Science and Biotechnology, 2: 35-39.

Lyons, E.M., Pote, J., Da Costa, M. and Huang, B. 2007. Whole-plant carbon relations and root respiration associated with root tolerance to high soil temperature for Agrostis grass. Environ. Exp. Bot, 59: 307-313.

Moud, A. M., and Maghsoudi, K. (2008). Salt stress effects on respiration and growth of germinated seeds of 
different wheat (Triticum aestivum L.) cultivars. World J. Agric. Sci, 4(3): 351-358.

Nyachiro, J. M., Clarke, F. R., DePauw, R. M., Knox, R. E., and Armstrong, K. C. (2002). Temperature effects on seed germination and expression of seed dormancy wheat. Euphytica, 126(1): 123-127.

Ortiz, R., Sayre, K. D., Govaerts, B., Gupta, R., Subbarao, G. V., Ban, T., Hodson, D., Dixon, J. M., Ortiz-Monasterio, J. I., and Reynolds, M. (2008). Climate change:Can wheat beat the heat? Agric. Ecosys. Environ, 126: 46-58.

Radi, A. A., Farghaly, F. A., and Hamada, A. M. (2013). Physiological and biochemical responses of salt-tolerant and salt-sensitive wheat and bean cultivars to salinity. Journal of Biology and Earth Sciences, 3(1): $72-$ 88.

Rivero, R. M., Ruiz, J. M., Garcia, P. C., Lopez-Lefebre, L. R., Sánchez, E., and Romero, L. (2001). Resistance to cold and heat stress: accumulation of phenolic compounds in tomato and watermelon plants. Plant Science, 160(2): 315-321.
Shaddad, M. A. K., HM, A. E. S., and Mostafa, D. (2013). Role of gibberellic acid $\left(\mathrm{GA}_{3}\right)$ in improving salt stress tolerance of two wheat cultivars. International Journal of Plant Physiology and Biochemistry, 5(4): 50-57.

Saba SJ, Kamal SA, Sadegh M, Avishan TT, Alireza G (2014). Effects of elevated temperatures on seed germination and seedling growth in three medicinal plants. Int. J. Agric. Crop Sci, 7(4):173-177.

Touchette, B.W., L.R. Iannacone, G.E. Turner, and A.R. Frank. (2007). Drought tolerance versus drought avoidance: A comparison of plant water relations in herbaceous wetland plants subjected to water withdrawal and repletion. Wetlands, 27: 656-667.

Turkyilmaz, B. (2012). Effects of salicylic and gibberellic acids on wheat (Triticum aestivum L.) under salinity stress. Bangladesh Journal of Botany, 41(1): 29-34.

$\mathrm{Xu}$, Q. and Huang, B. 2000a. Growth and physiological responses of creeping bentgrass to changes in shoot and root temperatures. Crop Sci, 40: 1365-1368

\section{How to cite this article:}

Neelambari, Ajay Kumar Singh and Shailesh Kumar. 2018. Effect of Individual and Combined Salinity and High Temperature Stress during Germination Stage of Different Wheat (Triticum aestivum L.) Genotypes. Int.J.Curr.Microbiol.App.Sci. 7(07): 1723-1730.

doi: https://doi.org/10.20546/ijcmas.2018.707.204 